\title{
Incidence of Shoulder Injury in Elite Wheelchair Athletes Differ Between Sports: A Critically Appraised Topic
}

\author{
Jessica R. Fairbairn and Kellie C. Huxel Bliven
}

\begin{abstract}
Clinical Scenario: Until recently, injury epidemiology data on elite Paralympic athletes were limited. Current data suggest high rates of shoulder injury in wheelchair athletes. Differences in shoulder injury rates between sports have not been reported in this population. Clinical Question: Is the incidence of shoulder injury in elite wheelchair athletes different between sports? Summary of Key Findings: Shoulder injury rates are high in elite wheelchair athletes, particularly in sports such as field events and fencing that require a stable base (eg, trunk, core control) from which to perform. Wheelchair racing requires repetitive motions that contribute to shoulder injuries, but rates are lower than field sports and fencing. Wheelchair curling and sledge hockey have low shoulder injury risk. Clinical Bottom Line: Shoulder injury rates vary based on sport in elite wheelchair athletes. In addition to incorporating shoulder complex specific rehabilitation for overuse shoulder injuries, clinicians should focus on core and trunk stabilization in elite wheelchair athletes competing in sports, such as field events and fencing. Strength of Recommendation: Grade C evidence exists that reports shoulder injury rates among elite wheelchair athletes differ based on sport participation.
\end{abstract}

Keyword: disabled training and conditioning, epidemiology, shoulder joint, Paralympic

\section{Clinical Scenario}

The inaugural Paralympic games were held in 1960, yet it was not until 1976 when injuries during the games were first documented. ${ }^{1}$ There are limited epidemiological data on disabled athletes due to inconsistent documentation, lack of definitions of injuries, reliance on self-reporting, and lack of web-based injury surveillance systems. Ferrara and Peterson ${ }^{1}$ conducted the first longitudinal study on 5 major elite paraevents using standardized procedures and equipment and reported injury rates in elite disabled athletes were comparable to elite able-bodied athletes. Their research also advocated for the development of standardized injury definitions, reporting more specific injury information (eg, wheelchair athletes by class and impairment), and data collection systems to enable higher quality epidemiological research of disabled athletes. ${ }^{1}$ Continued efforts by several organizations to address identified barriers led to standardized injury definitions and injury rate calculations, and the implementation of an online web-based injury and illness surveillance system at the 2012 London Paralympic Games. ${ }^{2,3}$ Establishing the infrastructure to support injury surveillance of elite disabled athletes facilitates consistent data collection and tracking methods across a variety of sports, body regions, and injuries. Information gained from injury surveillance enables researchers and clinicians to better understand injuries and the unique needs of this population, so more appropriate care can be provided. For example, wheelchair athletes present with unique demands and needs of their upper-extremity in daily life and sport, which may impact injury rates. It is important to know whether, how, and if sport type impacts shoulder injury rates, so appropriate steps can be implemented to restore upper-extremity function.

Fairbairn is with the Physical Therapy Department, Arizona School of Health Sciences, A.T. Still University, Mesa, AZ. Huxel Bliven is with the Department of Interdisciplinary Health Sciences, Arizona School of Health Sciences, A.T. Still University, Mesa, AZ. Fairbairn (jfairbairn@atsu.edu) is corresponding author.

\section{Clinical Question}

Is the incidence of shoulder injury in elite wheelchair athletes different between sports?

\section{Summary of Search, Best Evidence Appraised, and Key Findings}

- The literature was searched for studies that reported on shoulder injuries among elite wheelchair athletes who participate in parasports.

- The literature search returned 8 possible studies (4 prospective cohort, 3 retrospective cohort, and 1 systematic review study) related to the clinical question; 2 studies ${ }^{4,5}$ met the inclusion criteria and were included.

- While performing a hand search of references of included articles, 1 study met the inclusion criteria and was included. ${ }^{6}$

- The studies revealed that reports of shoulder injuries for elite wheelchair athletes had various incidence rates among 5 different parasports. ${ }^{4-6}$

- The shoulder is reported as the most common site of injury compared with other body regions among wheelchair athletes in 2 of the studies. ${ }^{4,5}$

- Seated throwing field athletes had higher shoulder injury incident rate compared with wheelchair racing. ${ }^{4}$

- The role that trunk control has on the risk of shoulder injury among wheelchair fencers may be an important consideration to mitigating injury. ${ }^{5}$

- Sledge hockey and wheelchair curling reported a higher risk of upper-extremity injury compared with other winter parasports (eg, alpine skiing, Nordic skiing, biathlon) ${ }^{6}$ 


\section{Clinical Bottom Line}

Evidence supports the high prevalence of overuse shoulder injuries in elite wheelchair athletes, while incidence rate of shoulder injuries is varied and dependent on sport. Seated field events were found to have the highest incidence rate of shoulder injury followed by wheelchair fencing. ${ }^{4,5}$ The required mechanics of seated field events and wheelchair fencing and its relation to the disruption in the kinetic chain due to poor core/trunk control may be a contributing factor to the overuse injury rate. Clinicians should develop injury prevention and treatment programs that restore core/ trunk control, thus promote optimal energy transfer through the kinetic chain, creating a more stable base from which fencing and throwing upper limb movements are generated. Additional research is needed to further investigate the biomechanics of individual sports and the upper-extremity compensations needed to participate in wheelchair athletes.

\section{Strength of Recommendation}

Grade $\mathrm{C}$ evidence exists that reports shoulder injury rates among elite wheelchair athletes differ based on sport participation.

\section{Search Strategy}

We performed a computerized search in April 2017 and again in October 2017. The following search terms in combination were put into the databases at once.

\section{Terms Used to Guide Search Strategy}

- Patient/Client Group: (Wheelchair OR adaptive OR Paraly* OR para-ath* OR disable*) AND (athlete)

- Intervention (or Assessment): not applicable

- Comparison: (track OR field OR basketball OR tennis OR skiing OR archery OR swimming OR rugby OR weight* OR sport OR compet*)

- Outcome(s): (prevalence OR epidemiology OR rate OR incidence) AND (injury OR pain OR chronic OR dysfunct* OR impair* OR disabil*) AND (shoulder OR upper extremity OR upper limb OR glenohumeral)

\section{Sources of Evidence Searched}

- The Cochrane Library

- PubMed

- CINAHL

- SPORTDiscus

- Additional resources obtained via review of reference lists and hand search

\section{Inclusion and Exclusion Criteria}

\section{Inclusion Criteria}

- Level 3 evidence or higher

- Studies that reported shoulder injury rates in elite wheelchair athletes. For the purposes of this study, "elite" was defined as individuals competing at a national or international level.
- Studies that investigated shoulder injury rates and/or pain among elite wheelchair athletes by sport.

- Studies that included able-bodied and wheelchair data but reported shoulder injury rates for each group separately

- Limited to English language

- Limited to the past 10 years (2007-2017)

\section{Exclusion Criteria}

- Studies that included patients whose injuries were related to daily function.

- Studies on disabled athletes, collectively, and did not separate out wheelchair athletes

- Studies that included but did not focus on athletes

\section{Results of Search}

Three relevant studies ${ }^{4-6}$ were located and categorized as shown in Table 1 (based on levels of evidence, Centre for Evidence-Based Medicine, 2011).

\section{Best Evidence}

The studies in Table 2 were identified as the best evidence and selected for inclusion in this critically appraised topic (CAT). These studies were selected because they were considered level 3 evidence or higher and reported shoulder injury rates by sport in elite wheelchair athletes.

\section{Implications for Practice, Education, and Future Research}

All 3 studies reviewed in this CAT addressed the incidence rate of shoulder injuries among elite wheelchair athletes, providing evidence to answer our clinical question of whether rates differ by sport. Wheelchair fencing and seated field events were at the highest risk for an overuse shoulder injury. While the same injury rate and risk values were reported differently in the studies, wheelchair fencing in athletes with poor trunk control had a shoulder injury rate of $13.6(3.4,17.8),{ }^{5}$ and wheelchair seated field events reported a rate of $15.8 \% .^{4}$ Wheelchair racing reported comparatively lower shoulder complex injury rates at 3.5\%.4 Wheelchair curling reported the lowest rate of shoulder complex injuries between sports at $0.11 \%{ }^{6}$ Sledge hockey reported only one traumatic shoulder injury, which was a glenohumeral dislocation. ${ }^{6}$ This is important information to consider when advising individuals on participation in the various wheelchair sports.

Table 1 Summary of Study Designs of Articles Retrieved

\begin{tabular}{llcl}
\hline $\begin{array}{l}\text { Level of } \\
\text { evidence }\end{array}$ & Study design & $\begin{array}{c}\text { Number } \\
\text { located }\end{array}$ & Reference \\
\hline 3 & Prospective cohort & 1 & $\begin{array}{l}\text { Chung et } \mathrm{al}^{5} \\
\end{array}$ \\
& Retrospective cohort & 2 & $\begin{array}{l}\text { Blauwet et } \mathrm{al}^{4} \\
\text { Webborn et } \mathrm{al}^{6}\end{array}$ \\
\hline
\end{tabular}




\begin{tabular}{ll}
\hline & Chung et $\mathbf{a l}^{\mathbf{5}}$ \\
\hline Study design & Prospective cohort \\
Participants & 10 elite AFs: age $=27.0(5.5) \mathrm{y}$ \\
& 14 WFs: age $=28.6(6.8) \mathrm{y} ; \mathrm{n}=7$ category \\
& A (good trunk control), $\mathrm{n}=7$ category B \\
& (no active trunk control) \\
& Inclusion criteria: members of Hong Kong \\
& National Squad, available for monthly \\
& interviews over a 3-y time span \\
& No exclusion criteria were reported.
\end{tabular}

Intervention Three physiotherapists conducted the investigated monthly interviews between November 2006 and October 2009.

A standardized injury recording form was used.

$\mathrm{AF}$ and $\mathrm{WF}$ responded to questions on training duration, match duration, and self-reported injury characteristics.

If an injury was self-reported, it was examined and diagnosed by a registered physiotherapist and an orthopedic specialist.

Outcome measure(s)

Main findings

(1) Number of injuries between $\mathrm{AF}$ and WF

(2) Number of injuries between WF category A and category $\mathrm{B}$

(3) RR between AF and WF in relation to the nature, location, and severity

(4) RR between WF category A and category $\mathrm{B}$ in relation to the nature, location, and severity.

62 injuries reported among AFs, 95 injuries reported among WFs.

Competition reported higher injuries compared with training for both $\mathrm{AF}$ and

WF. These injuries were considered acute and traumatic.

LE injuries (43) were reported more common in AFs, whereas UE injuries (70) were more common in WFs.

WFs are more at risk for minor injuries $(\mathrm{RR}=2.4$; 95\% CI, 1.6-3.6), muscle strain $(\mathrm{RR}=2.2 ; 95 \% \mathrm{CI}, 1.3-3.6)$, shoulder injuries $(\mathrm{RR}=13.6$; 95\% CI, 3.4-17.8), and elbow injuries $(\mathrm{RR}=5.9 ; 95 \% \mathrm{CI}$, 2.5-17.2).

WF category $B$ reported more injuries than WF category A (55 injuries and 40 injuries, respectively).

WF category A had higher risk of muscle strain $(\mathrm{RR}=1.83 ; 95 \% \mathrm{CI}, 1.1-3.3)$ and shoulder injury $(\mathrm{RR}=4.97 ; 95 \% \mathrm{CI}$, 1.8-16.9) compared with WF category B. Extended absence of training/competition for $\mathrm{AF}$ was due to various $\mathrm{LE}$ orthopedic conditions while WF, especially category $\mathrm{B}$, shoulder injuries were responsible for time loss.

\section{Blauwet et $\mathrm{al}^{4}$}

Retrospective cohort ${ }^{\mathrm{a}}$

977 elite paraathletes (males and females) from 138 countries

Inclusion criteria: paraathlete at the London 2012 Paralympics who competed in the sport of track and field No exclusion criteria were reported.

All participants were monitored over the 10-d competition period for injury. Injury reports were collected daily via WEB-IISS by the individual team's medical staff and EMDCS by LOCOG medical staff.

International Paralympic Committee athlete database provided the athlete's impairment and event discipline.

(1) IR by impairment, event discipline, sex, and age

(2) Characteristics of paraathlete track and field injuries

(3) Role of risk factors, such as sex, age, discipline, and impairments on IR

216 injuries were reported: 95 injuries/497 athletes in track and 121 injuries/480

athletes in field.

Overall IR $=22.1$

Track:

- Impairment: IR = 63.1 for ambulatory athletes with amputations, visual impairments, and $\mathrm{CP}$

- $\mathrm{IR}=10.6$ for wheelchair racing athletes

- Sex: 78 injuries/355 male athletes and 17 injuries/142 female athletes

- Age: no significant difference in IR between ages

Field:

- Impairment: IR = 20.5 for ambulatory throwing athletes

- $\mathrm{IR}=23.7$ for seated throwing athletes

- Sex: no significant difference in IR between male and female

- Age: no significant difference in IR among athletes aged 13-25, 26-34, and $>35 \mathrm{y}$

Wheelchair/seated athletes:

- 57 injuries/319 athletes

- Overall IR = 17.9

- Field athletes IR=23.7; wheelchair racing $\mathrm{IR}=10.6$

- Competition IR=7.5 and training $\mathrm{IR}=2.2$

- Location: $19.3 \%$ shoulder/clavicle; $3.5 \%$ shoulder/clavicle for wheelchair racing; and $15.8 \%$ shoulder clavicle for seated throwing

\section{Webborn et $\mathrm{al}^{6}$}

Retrospective cohort ${ }^{\mathrm{a}}$

118 elite sledge hockey athletes; 51 elite wheelchair curling athletes

Inclusion criteria: paraathlete at the

Vancouver 2010 Paralympics who consented to the survey, data that were collected between the opening and closing of the Paralympic Villages

Exclusion criteria: any illness that were reported and any athlete who did not want to participate in the survey

Athletes self-reported on the "occurrence" of the injury.

Injuries were documented using paper and electron forms at the games' various polyclinics.

These forms were then submitted and reviewed by the research team.

Cross-check of questionnaires was performed manually to ensure no duplications.

(1) IR

(2) IR ratios

(3) Incidence proportion reported overall and by sport

(4) Descriptive data on injury onset, injured body part, and injury type

Ice sledge hockey:

- IP: 40 athletes (33.9\%) reported injuries

- Age: mean age $=33$

- Type: $40 \%$ acute injuries and $60 \%$ overuse injuries

- Onset: 21 (52.5\%) injuries occurred during competition, 7 (17.5\%) injuries occurred during training at the games, $4(10 \%)$ injuries occurred prior to the games related to sport, $4(10 \%)$ did not report a time, and $4(10 \%)$ injuries occurred prior to the games but were not sport related

- Location: 19 (47.5\%) UE, 14 (35\%) spine related, 4 (10\%) LE, 2 (5\%) head, and $1(2.5 \%)$ thoracic

- Description: 12 (30\%) muscle strains, $4(10 \%)$ ligament sprains, $3(7.5 \%)$ fractures, $2(5 \%)$ contusions, $2(5 \%)$ lacerations, $1(2.5 \%)$ glenohumeral dislocation, and $16(40 \%)$ less welldefined injuries (ie, mechanical LBP, scapulothoracic dysfunction, impingement hypertonicity) Wheelchair curling:

- $9(18 \%)$ athletes reported minor musculoskeletal injuries

- Type: 9 (18\%) were overuse injuries

- Onset: 5 of the 9 injuries were prior to the games but were not sport related

- Location: spine: 3 cervical, 1 thoracic, 2 lumbar, UE: 1 shoulder, 1 elbow, 1 wrist

- Description: all injuries were overuse/ strain 
Table 2 (continued)

\begin{tabular}{llll}
\hline & ${\text { Chung et } \mathbf{~ a l ~}^{\mathbf{5}}}$ & ${\text { Blauwet } \mathbf{~ e t ~} \mathbf{a l}^{\mathbf{4}}}^{\text {Webborn }_{\text {et }} \mathbf{a l}^{\mathbf{6}}}$ \\
\hline $\begin{array}{l}\text { Level of } \\
\text { evidence }\end{array}$ & 3 & 3 & 3 \\
$\begin{array}{l}\text { Validity score } \\
\text { (if } \text { N/A }\end{array}$ & N/A & N/A
\end{tabular}

(if applicable)

Conclusion
AFs have a higher risk for LE injuries, whereas WFs have a higher risk for UE injuries.

Truck control may play a role in risk of injury for WF.

This article can be used to further research on injury incidence and biomechanics as they relate to injury among both abled and disabled athletes.
Ambulatory athletes are at greater risk for LE injuries, whereas wheelchair/seated athletes are at greater risk for UE injury in particular throwing athletes.

This article can be used to further research on injury prevention and tailored programs based on incidence and anatomical region.
Sledge hockey athletes have a higher risk of overuse UE injuries. Wheelchair curling athletes are at a greater risk for overuse/strain spinal injuries.

Future research is needed to examine the biomechanics of injury to assist in comprising an injury prevention program.

\begin{abstract}
Abbreviations: \%, percent of total injuries; AF, able-bodied fencer; CI, confidence interval; CP, cerebral palsy; EMDCS, electronic medical data capture system; IP, incidence proportion (injuries per 100 athletes); IR, incidence rate (injuries per 1000 athlete days); LBP, low back pain; LE, lower-extremity; LOCOG, London Organizing Committee of the Olympic and Paralympic Games; N/A, not applicable; RR, relative risk of injury (95\% CI); UE, upper-extremity; WEB-IISS, web-based injury and illness surveillance system; WF, wheelchair fencer.

${ }^{\mathrm{a}}$ For the purpose of this study, information pertaining specifically to the clinical question is presented in the table. The articles by Blauwet et $\mathrm{al}^{4}$ and $\mathrm{Webborn}$ et al ${ }^{6}$ reported on multiple areas of the Paralympics and were not included in the table.
\end{abstract}

Until recently, it has been difficult to compare results of previous parasports epidemiological studies due to the overall lack of research and the inconsistency of injury reports. However, previous studies on wheelchair sports have consistently reported high rates of overuse upper-extremity injuries, specifically to the shoulder. ${ }^{1,7,8}$ Although the most recent epidemiological study using data collected at the Rio Paralympics did not meet our study inclusion criteria, it reiterates findings that the shoulder is the most susceptible body part to overuse injury. ${ }^{9}$ Overuse shoulder injuries in wheelchair athletes are commonly reported as soft-tissue injuries due to repetitive nature. ${ }^{1,7,8}$ This mechanism typically refers to the repetitive motions associated with wheelchair propulsion as seen in racing sports. ${ }^{8}$ Although repetitive wheelchair propulsion may contribute to overuse shoulder injuries, more recent research suggests this mechanism may not be the leading cause of overuse shoulder injuries in this population.

Current research suggests that overuse shoulder injuries occur due to a deficit in the kinetic chain. ${ }^{5}$ It is speculated that the combination of performing the sport seated and the lack of power generated by the lower-extremity creates an unstable base, further challenging the body to produce a powerful upper-extremity movement. Deficits along the kinetic chain lead to greater compensations at the upper-extremity and over time results in an overuse injury. This concept is further supported by the fact that the wheelchair group with decreased trunk control displayed a higher prevalence of overuse shoulder injuries compared with the wheelchair group with trunk control. ${ }^{5}$ Wheelchair fencing reported the second highest injury incident rate out of 22 sports at the Rio Paralympics. ${ }^{9}$ Athletes who utilize a wheelchair while competing in sports (eg, wheelchair rugby, wheelchair basketball, wheelchair tennis) were among the top 8 sports with the highest injury rate. ${ }^{9}$ This further supports the concept of an unstable base contributing to injury. Determining the injury to be caused by the repetitive deficits in kinetic chain rather than a single repetitive motion is a unique aspect and finding. It is important for clinicians to consider this as another common mechanism of overuse shoulder injuries when working with paraathletes who compete seated.

The findings of this CAT demonstrate the need for clinicians to have a greater understanding of risk factors that can and cannot be modified specific to the elite wheelchair athletes. Clinicians should be aware of the type of injury not only if covering a parasporting event but also as wheelchair athletes seek medical treatment in the clinic. The utilization of injury incidence rates among the different sports and anatomical regions in conjunction with the risk factors is crucial in the implementation of prevention and rehabilitation programs.

Future research should include investigation of injury rates among and within the various disability classification groups used in parasports to provide more specific information on injuries as they relate to specific disabilities. ${ }^{1}$ Research should also include a more detailed description of the mechanism of injury to help establish injury patterns, which can be utilized to develop prevention programs and preparticipation evaluations. ${ }^{1}$ In addition, there is a need for further research on this topic that involves injuries reported throughout training and not just at single events. This will provide a more extensive understanding of overuse injury incidence rates and its effect among wheelchair athletes. This CAT should be reviewed in 2 years or when additional best evidence becomes available to determine whether additional best evidence has been published that may change the clinical bottom line for the research question posed in this review.

\section{References}

1. Ferrara MS, Peterson CL. Injuries to athletes with disabilities: identifying injury patterns. Sports Med. 2000;30(2):137-143. PubMed ID: 10966152 doi:10.2165/00007256-200030020-00006

2. Derman W, Schwellnus M, Jordaan E, et al. Illness and injury in athletes during the competition period at the London 2012 Paralympic Games: development and implementation of a web-based surveillance system (WEB-IISS) for team medical staff. Br J Sports Med. 2013;47(7):420-425. PubMed ID: 23537560 doi:10.1136/bjsports2013-092375

3. Willick SE, Webborn N, Emery C, et al. The epidemiology of injuries at the London 2012 Paralympic Games. Br J Sports Med. 2013; 47(7):426-432. PubMed ID: 23515713 doi:10.1136/bjsports-2013092374 
4. Blauwet CA, Cushman D, Emery C, et al. Risk of injuries in Paralympic track and field differs by impairment and event discipline: a prospective cohort study at the London 2012 Paralympic Games. Am J Sports Med. 2016;44(6):1455-1462. PubMed ID: 26920432 doi:10.1177/0363546516629949

5. Chung WM, Yeung S, Wong AY, et al. Musculoskeletal injuries in elite able-bodied and wheelchair foil fencers-a pilot study. Clin J Sport Med. 2012;22(3):278-280. PubMed ID: 22430329 doi:10. 1097/JSM.0b013e31824a577e

6. Webborn N, Willick S, Emery CA. The injury experience at the 2010 Winter Paralympic Games. Clin J Sport Med. 2012;22(1):3-9. PubMed ID: 22222596 doi:10.1097/JSM.0b013e318243309f
7. Fagher K, Lexell J. Sports-related injuries in athletes with disabilities. Scand J Med Sci Sports. 2014;24(5):e320-e331. PubMed ID: 24422719 doi:10.1111/sms.12175

8. Taylor D, Williams T. Sports injuries in athletes with disabilities: wheelchair racing. Paraplegia. 1995;33(5):296-299. PubMed ID: 7630659 doi:10.1038/sc.1995.67

9. Derman W, Runciman P, Schwellnus M, et al. High precompetition injury rate dominates the injury profile at the Rio 2016 Summer Paralympic Games: a prospective cohort study of 51198 athlete days. Br J Sports Med. 2018;52(1):24-31. PubMed ID: 29030389 doi:10. 1136/bjsports-2017-098039 